\title{
Modeling slips and nucleation processes at the deeper part of the seismogenic zone
}

\author{
Bunichiro Shibazaki ${ }^{1}$, Norio Shigematsu ${ }^{2}$, and Hidemi Tanaka ${ }^{3}$ \\ ${ }^{1}$ International Institute of Seismology and Earthquake Engineering, Building Research Institute, 1 Tatehara, Tsukuba 305-0802, Japan \\ ${ }^{2}$ Geological Survey of Japan, AIST, AIST Tsukuba Central 7, 1-1 Higashi, Tsukuba 305-8567, Japan \\ ${ }^{3}$ Department of Earth and Planetary Sciences, The University of Tokyo, 7-3-1 Hongo, Tokyo 113-0033, Japan
}

(Received July 12, 2004; Revised November 25, 2004; Accepted November 30, 2004)

\begin{abstract}
Important issues with regard to the generation processes of large inland earthquakes include how the stress concentrates and how nucleation starts in the deeper part of the seismogenic zone prior to the mainshock. We propose a model of earthquake generation processes that uses a constitutive law combining friction and flow processes. Using this law, we can represent fault behavior in which frictional slip coexists with flow processes at the frictional-viscous transition zone. We consider a limitted region where viscous deformation is high along the frictional-viscous transition zone, and investigate the role of this region in the nucleation process. During the interseismic period, slip velocity due to flow is much larger than frictional slip velocity in the region of low viscosity in the deeper part of the seismogenic zone. Large slip due to flow in this region is thought to cause stress to concentrate in the surrounding regions, and nucleation starts just above the low-viscosity region. Our numerical simulations indicate that the location of the nucleation process is determined by the nonuniform distribution of the depth of the frictional-viscous transition zone.
\end{abstract}

Key words: Nucleation process, a constitutive law combining friction and flow, frictional-viscous transition, low-viscosity region.

\section{Introduction}

It is widely recognized that the lithosphere consists of three different regimes in terms of their deformation style: the brittle regime controlled by friction law, transition from brittle regime to viscous regime, and the viscous regime controlled by dislocation creep (e.g., Chester, 1995). In the frictional regime, there exist three different regions: the unstable seismogenic region, the unstable-stable transition, and the deeper stable region. At the deeper frictionally stable region, plastic deformation of asperities on frictional surfaces is thought to be dominant (e.g. Sholz, 1990). This region can be regarded as a part of the brittle-viscous transition zone. At the viscous region below this region, bulk viscous deformation of rock is thought to occur in shear zones.

This structure of the lithosphere plays a significant role in the control of earthquake generation processes. The scenario of the generation processes of inland earthquakes is as follows (e.g., Iio and Kobayashi, 2002; Iio et al., 2002): (I) a localized shear zone exists at the deeper extension of the seismogenic fault in the lower crust, (II) a stationary slip proceeds at the deeper extension of the seismogenic fault during the interseismic period which results in stress concentrating at the bottom of the seismogenic zone, and finally (III) the slip accelerates widely in and below the deeper part of the seismogenic zone.

Copy right(c) The Society of Geomagnetism and Earth, Planetary and Space Sciences (SGEPSS); The Seismological Society of Japan; The Volcanological Society of Japan; The Geodetic Society of Japan; The Japanese Society for Planetary Sciences; TERRAPUB
To investigate the mode of shear localization in the lower crust (e.g., Tanaka et al., 2002), we need to investigate volumetric deformation by using a method such as the finite element method. For this purpose, Garatani et al. (2005) developed a finite element code for non-linear visco-elasticity, plasticity, and faulting.

On the other hand, to model the processes of slip acceleration and nucleation, we need to investigate the fault model by using frictional constitutive laws. In this case, a boundary integral equation method is widely used. In previous studies (e.g. Tse and Rice, 1986; Kato and Hirasawa, 1997), to model the slip processes below the seismogenic zone, the frictional property was simply changed from velocity-weakening to velocity-strengthening. However, frictional behavior in the transition zone seems to be more complex. Shimamoto (1986) examined the dependence of steady state friction on slip velocity $v$ by using halite around the unstable-stable transition zone. He found that at very low velocity $\left(v \ll 10^{-6} \mathrm{~m} / \mathrm{s}\right)$ the steady state friction $\tau_{s s}$ increases with slip velocity $v$; at low velocity $\left(v \approx 10^{-6} \mathrm{~m} / \mathrm{s}\right), \tau_{s s}$ decreases with $v$; and at high slip velocity $\left(v \gg 10^{-6} \mathrm{~m} / \mathrm{s}\right), \tau_{s s}$ increases with $v$. This experimental study implies that, around the brittle-viscous transition zone, there are three different mechanisms, depending on the slip velocity: (I) the flow process is dominant at very low slip velocity; (II) velocity-weakening friction is dominant at low slip velocity; and (III) velocity-strengthening friction is dominant at high slip velocity.

We have two possible kinds of constitutive laws for the frictional-viscous transition zone. The first law is the con- 


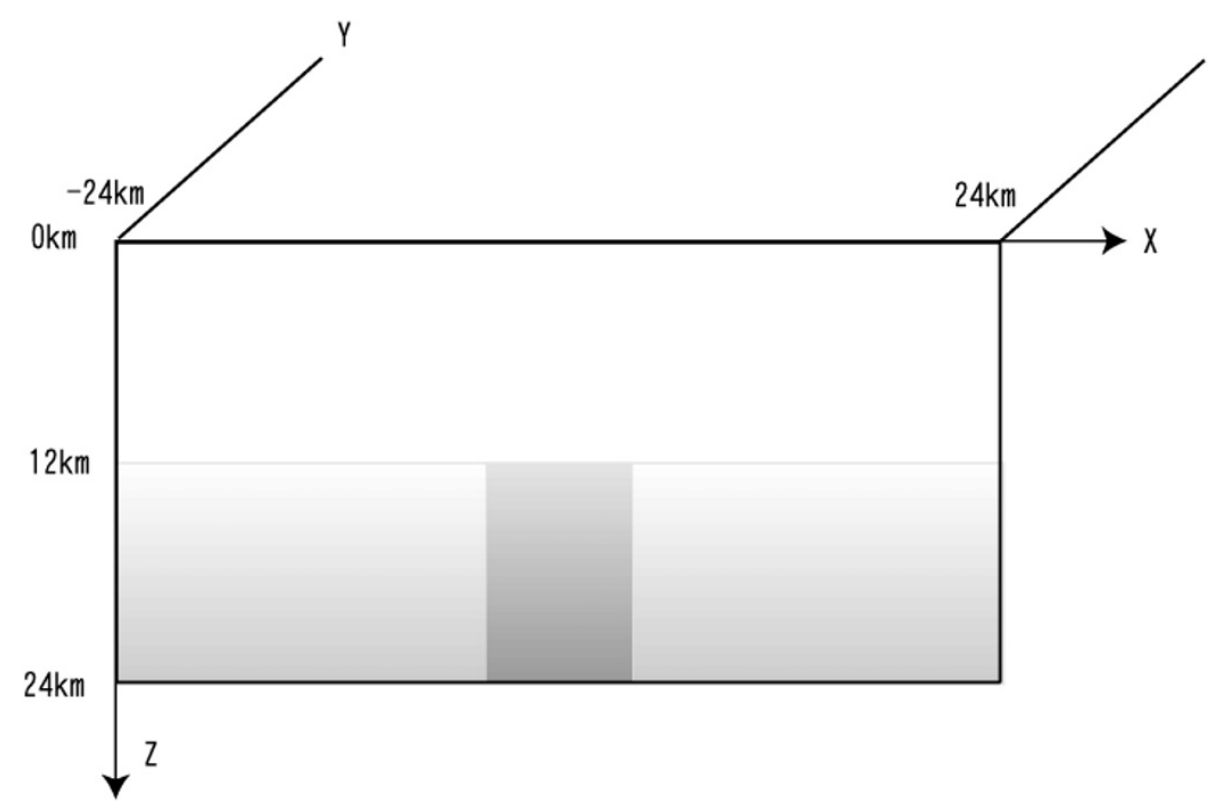

Fig. 1. Strike-slip fault plane in an elastic-space. The length and width of the fault plane are taken to be 48 and $24 \mathrm{~km}$, respectively. Cell size is taken to be $400 \mathrm{~m}$. The depth of the brittle-viscous transition is set to be around $12 \mathrm{~km}$. A low-viscosity region is located at $-8(\mathrm{~km}) \leq x \leq 8(\mathrm{~km})$, and $z \geq 12(\mathrm{~km})$.

stitutive law combining flow process and friction. Using this law, around the transition zone from frictional regime to viscous regime, at very low slip velocity viscous flow is dominant and at high-slip velocity frictional slip is dominant. Shibazaki et al. (2002) examined 1-D and 2-D models by using a constitutive law combining friction and flow processes. The second law is the constitutive law that exhibits velocity weakening at low slip velocity and velocity strengthening at high slip velocity. Using this law, slip acceleration below the deeper part of the seismogenic zone can been reproduced (Shibazaki and Iio, 2003; Kato, 2003).

In the present study, given the spatial variation of parameters in the first law on the fault in 3-D elastic half-space, we investigate the role of the frictional-viscous transition zone on the nucleation process of large earthquakes. Recently, Shigematsu et al. (2003) investigated deformation styles along the Hatagawa fault zone and proposed a model in which a highly deformed region exists along the frictionalviscous transition zone. We discuss the role of this highly deformed region from a theoretical point of view.

\section{Model of Nucleation Using Constitutive Law Combining Friction and Flow}

\subsection{Constitutive law}

Multi-mechanism friction laws have been proposed by several authors (e.g., Chester 1995; Reinen, 2000). Shibazaki et al. (2002) investigated a fault model in which a rate- and state-dependent friction law coexists with a power law creep that has a threshold stress. The constitutive equation which they used is the following:

$$
\tau=\tau_{f}=\tau_{d}
$$

where $\tau_{f}$ is frictional stress and $\tau_{d}$ is shear stress due to flow processes. Total fault displacement is the sum of frictional slip and slip due to flow processes,

$$
u=u_{f}+W \varepsilon_{d}
$$

where $u_{f}$ is frictional slip, $W$ is the width of the fault zone, and $\varepsilon_{d}$ is the strain due to the flow processes. Frictional stress is written as:

$$
\tau_{f}=\sigma_{n}^{e f f} \mu,
$$

where $\sigma_{n}^{e f f}$ is the effective normal stress defined as difference between lithostatic pressure and pore-fluid pressure, and $\mu$ is the frictional coefficient.

There are two kinds of flow processes: power law dislocation creep and exponential law creep due to thermal activation process in which the strain rate increases exponentially with increasing shear stress. At the greater depth in the lower crust, power law dislocation creep is thought to be dominant. The physical interpretation of the direct effect in a rate- and state-dependent friction law is thermal activation creep between microasperities of contacting surfaces (e.g. Nakatani, 2001). Therefore, exponential law creep due to thermal activation process is thought to be included in a rate- and state-dependent friction law. In the present study we will consider power law creep with the threshold stress $\tau_{t h}$, which obeys the following function:

$$
\tau_{d}=\tau_{t h}+a_{\text {flow }}\left(\dot{\varepsilon}_{d}\right)^{1 / n} \exp (Q / n R T) .
$$

where $a_{\text {flow }}$ is the constant, $Q$ is the activation energy, $n$ is the power law exponent and $T$ is temperature. In some cases, a threshold stress (internal stress) $\tau_{t h}$ is required to properly explain the experimental results (e.g. Poirier, 1985).

There are various forms of the frictional constitutive law, such as the Dieterich-Ruina friction law and the RuinaDieterich friction law (e.g. Marone, 1998). It would take 
(a)

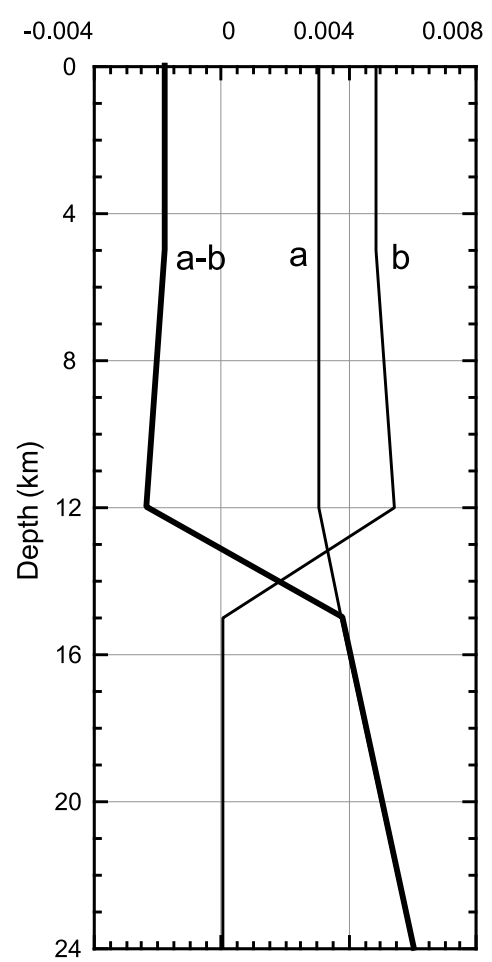

(b) Critical weakening displacement $(\mathrm{m})$

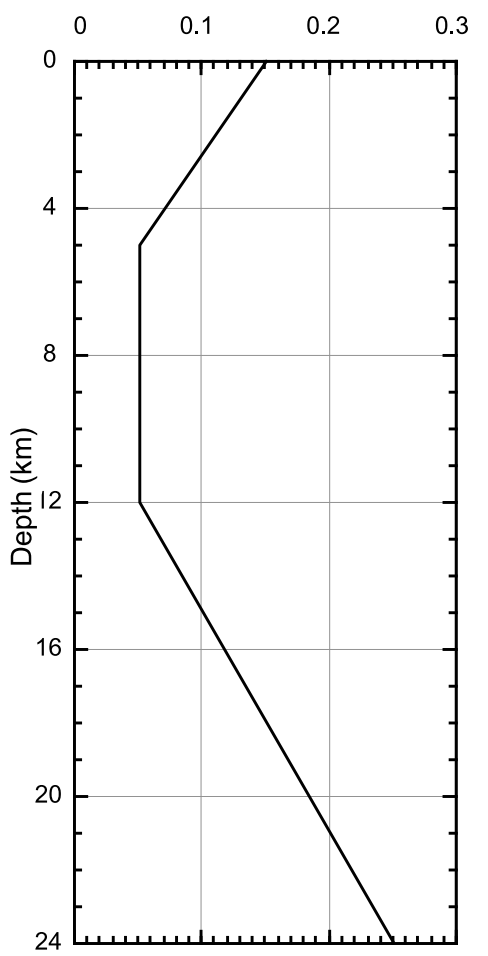

(c) Pore pressure (MPa)

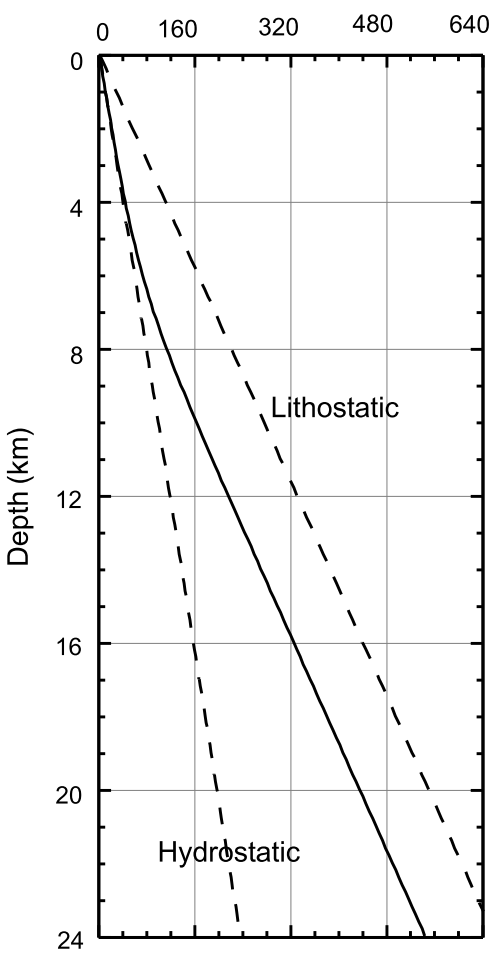

Fig. 2. Depth-distribution of constitutive law parameters $a, b$, and $a-b$ (a), critical weakening displacement $D_{c}$ (b), and pore fluid pressure $P_{f}$ (c).

too long for us to calculate fault slip using the constitutive law combining flow law and a Dieterich-Ruina constitutive law. Therefore, we use the Ruina-Dieterich friction law, as expressed by the following equations:

$$
\tau_{f r}=\sigma_{n}^{e f f}\left\{\mu_{*}+a \ln \left(v / \nu_{*}\right)+b \Psi\right\}
$$

where $\mu_{*}$ is the base friction for steady-state slip at reference velocity $\nu_{*}, v$ is a slip velocity, and $\Psi$ is a state variable. In the Ruina-Dieterich friction law, the state variable $\Psi$ can be written as

$$
d \Psi / d t=-v / D_{c}\left\{\Psi+\ln \left(v / v_{*}\right)\right\}
$$

where $D_{c}$ is a characteristic displacement scaling the evolution of the state variable.

\subsection{Tectonic loading process}

Let us consider a quasi-static analysis that assumes a vertical strike-slip fault in 3-D elastic half-space, as illustrated in Fig. 1. The fault plane is located on the $y=0$ plane in the Cartesian coordinate system $(x, y, z)$. The free surface is located on the $z=0$ plane. A fault plane is divided into rectangular cells with a length $\Delta x$ of and $\Delta z$. The $i j$-cell occupies the region $(i-1) \Delta x \leq x \leq i \Delta x(i=1, \ldots, M)$, $(j-1) \Delta z \leq z \leq j \Delta z(j=1, \ldots, N)$. The slips are assumed to be constant within each cell. For simplicity, we allow slips only in the $x$ direction. The shear stress $\tau_{i, j}$ on the $i j$-cell on the fault is accumulated by the delay of the fault slip $\mu_{i, j}$ from the long-term average slip of the fault,

$$
\tau_{i, j}=\sum_{i_{S} j_{S}} k_{i-i_{S}, j-j_{S}}\left(V_{l} t-u_{i_{S}, j_{S}}\right)-\frac{G}{2 \beta} \frac{d u_{i, j}}{d t},
$$

where $V_{l}$ is the long-term average slip velocity of the fault, $G$ is the rigidity, $\beta$ is the shear wave velocity, and $k_{i-i_{S}, j-j_{S}}$ is the elastostatic kernel, which is the stress at the center of the $i j$-cell caused by the uniform slip over a rectangular dislocation of the $i_{S} j_{S}$-cell. The first term represents tectonic loading due to the long-term average slip of the fault. The second term represents the seismic radiation damping introduced by Rice (1993) in order to consider the approximate effect of inertia. We used the program coded by Iwasaki and Sato (1979) to calculate the kernels. We solve Eqs. (1), (2), and (4)-(7) using the Runge-Kutta method with an adaptive time step size control which Kato and Hirasawa (1997) originally used.

\section{Numerical Analysis}

Recently, Shigematsu et al. (2003) investigated deformation styles along the Hatagawa fault zone and proposed a model in which a highly deformed region exists along the frictional-viscous transition zone. It is thought that stress concentrates around this region and that the nucleation of dynamic rupture starts there. We consider the region where viscous deformation is high along the frictional-viscous transition zone, and investigate the role of this region in the nucleation process.

We used the same depth distributions of constitutive law parameters as those used by Shibazaki et al. (2002). The depth distribution of constitutive law parameters $a, b, a-b$, $D_{c}$, and $P_{f}$ are shown in Fig. 2. The effective normal stress is calculated from the difference between lithostatic pressure and pore-fluid pressure $P_{f}$ in Fig. 2(c). We set the long-term average slip velocity $V_{l}$ of the fault to be 0.5 $\mathrm{cm} /$ year.

First, we consider the case (Case 1) in which a lowviscosity region is located at the center of the fault zone 

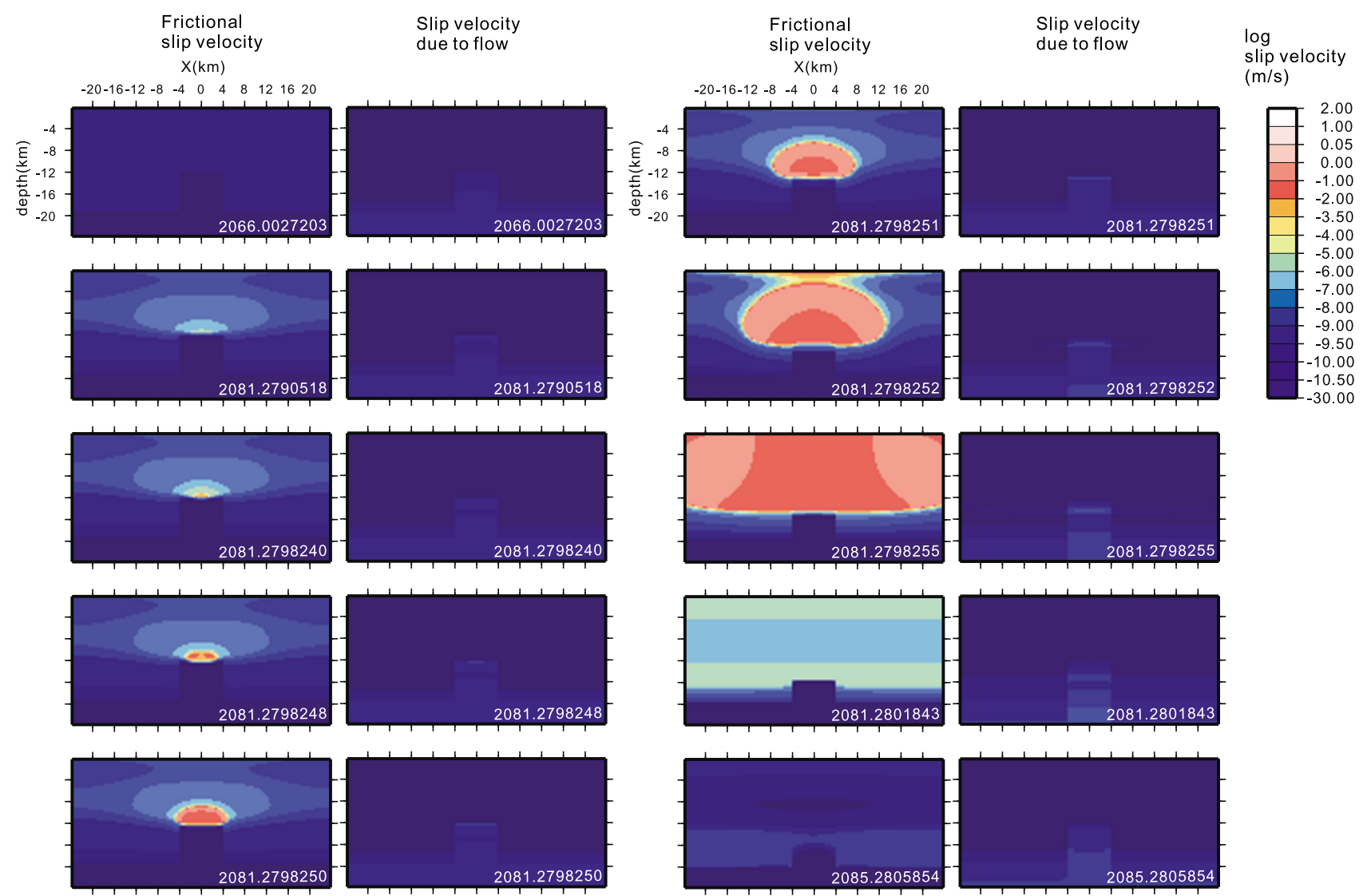

Fig. 3. Changes of frictional slip velocity distribution (left) and slip velocity distribution due to flow (right) with time for case 1 . Numbers shown in each panel is the time in years. A low-viscosity region is located at $-8(\mathrm{~km}) \leq x \leq 8(\mathrm{~km})$, and $z \geq 12(\mathrm{~km})$.

and below a depth of $12 \mathrm{~km}$. We set the value of $Q$ to be $131.75 \mathrm{~kJ} / \mathrm{mol}$ at the low-viscosity region. In the surrounding region we set the values of the threshold stress $\tau_{t h}$, the activation energy $Q, n$, and $W$ are set to be $0.9 \mu_{*} \sigma_{n}^{\text {eff }}, 155$ $\mathrm{kJ} / \mathrm{mol}, 3$, and $10 \mathrm{~m}$ to make the depth of brittle-viscous transition about $12 \mathrm{~km}$. The depth distribution of temperature given by Iio and Kobayashi (2002) is used.

Frictional slip velocity distributions and slip velocity distributions due to flow at each time step from the nucleation process to the cessation of rupture are shown in Fig. 3. During the interseismic period, slip velocity due to flow is much larger than frictional slip velocity at the low-viscosity region (Fig. 4(a)). In the surrounding region, frictional slip velocity is dominant during the interseismic period (Fig. 4(b)). However, slip velocity due to flow in the lowviscosity region (Fig. 4(a)) is larger than frictional slip velocity in the surrounding region (Fig. 4(b)) during this period. Thus, the large amount of slip due to flow in the low-viscosity region during the interseismic period seems to cause the stress to concentrate in the surrounding regions, and nucleation starts just above the low-viscosity region. During the coseismic period (Fig. 4(a)), frictional slip velocity in the low-viscosity region increase rapidly and then decreases to a very low value. Coseismic rupture propagates to the deeper part of the fault zone, where power law creep is dominant during the interseismic period (Fig. 4(c)). In a case where parameters of constitutive law are uniform in the horizontal direction, nucleation tends to start at the center of or the edge of the fault zone. To confirm that the location of the nucleation zone is determined by the location of the low-viscosity region, we change the location of the low-viscosity region (Case 2). The location of the nucleation zone is changed and nucleation starts just above the low-viscosity region (Fig. 5(a)).

The pore-fluid pressure also controls the depth of the brittle-viscous transition. Shear stress is in proportion to the effective normal stress $\sigma_{n}-P_{f} ; \tau_{f}=\left(\sigma_{n}-P_{f}\right) \mu$. Shear stress decreases with the increase in the pore-fluid pressure. If there is a region of high pore-fluid pressure in the deeper part of the seismogenic zone, the depth of the brittleviscous transition zone is thought to increase. In the case of the 1995 Hyogo-ken Nanbu earthquake, the existence of high-pressure fluid around the nucleation zone has been pointed out by Zhao et al. (1996). The nucleation area of the Hyogo-ken Nanbu earthquake was significantly deeper than the cut-off depth of seismicity in the rupture area of the northeastern section. We examine a case (Case 3 ) in which a region of high pore-fluid pressure exists at the center of the fault zone and below a depth of $12 \mathrm{~km}$. In this region, $40 \mathrm{MPa}$ is added to the fluid pressure distribution shown in Fig. 2(c). The values of the threshold stress $\tau_{t h}$, the activation energy $Q, n$, and $W$ are set to be $0.9 \mu_{*} \sigma_{n}^{\text {eff }}, 180$ $\mathrm{kJ} / \mathrm{mol}, 3$, and $10 \mathrm{~m}$. Figure 5(b) shows the frictional slip velocity distributions and the slip distributions due to flow just before dynamic rupture. The nucleation zone is located just above the region of high pore-fluid pressure. 
(a)

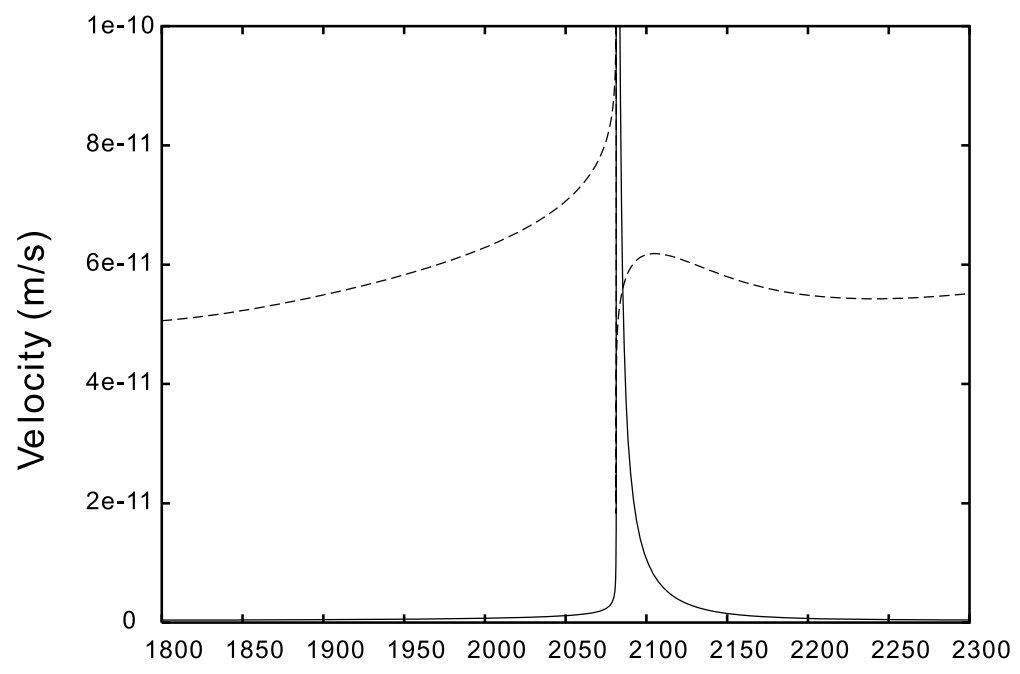

(b)

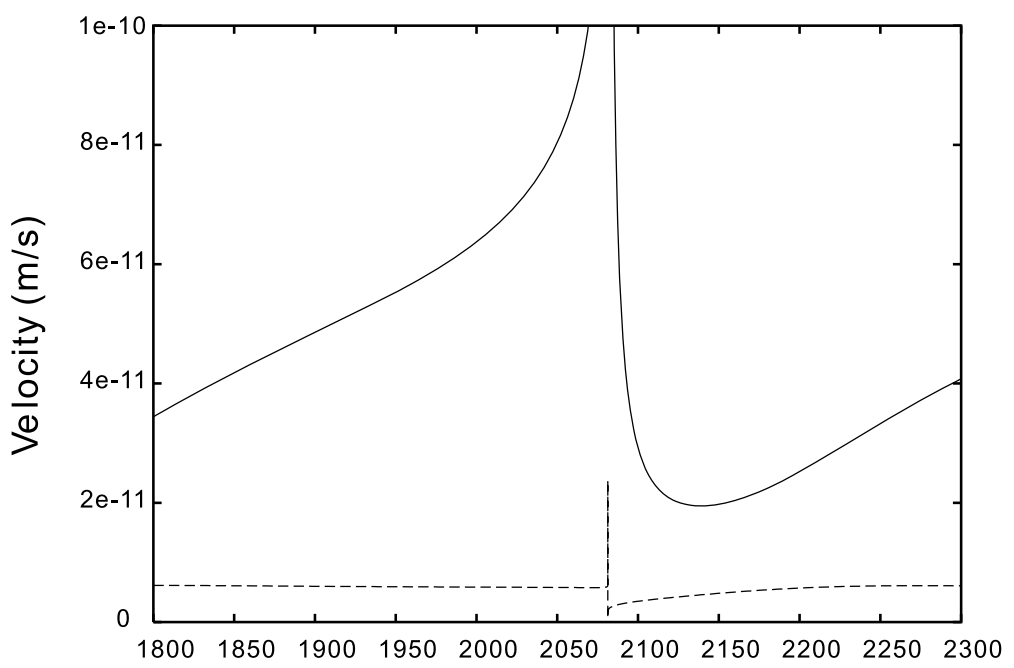

(c)

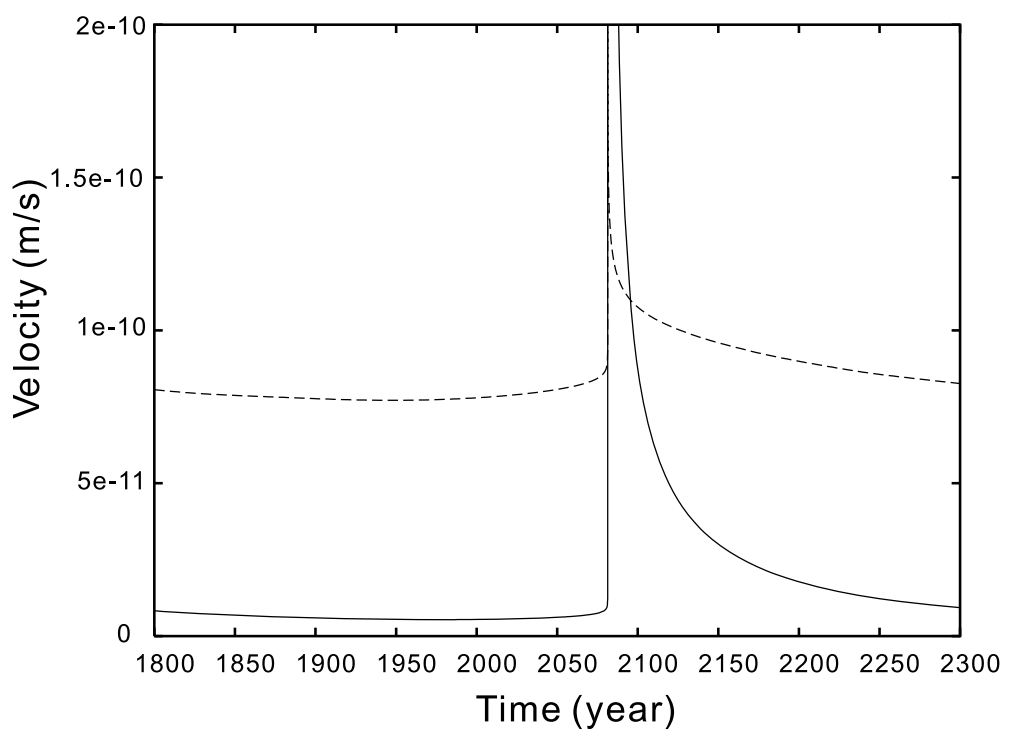

Fig. 4. Time changes in slip velocity due to flow (dashed line) and frictional slip velocity (solid line) at points $(x, z)=(-0.2 \mathrm{~km}, 12.2 \mathrm{~km})($ a), $(x, z)=(-12.2 \mathrm{~km}, 12.2 \mathrm{~km})(\mathrm{b}),(x, z)=(-0.2 \mathrm{~km}, 14.2 \mathrm{~km})(\mathrm{c})$.

\section{Discussion}

4.1 Slip and nucleation processes at the deeper part of the seismogenic zone

Ito (1990) examined the initiation process of several large inland earthquakes and found that they start at the deepest portion of the seismogenic layer and where sharp changes occur in the depth of the seismic-aseismic boundary. The depth of the seismic-aseismic boundary corresponds to the depth of the transition zone from frictional unstable zone to viscous zone. Observations by Ito (1990) suggested that the 

Frictional
slip velocity
(a) Case2
$\mathrm{X}(\mathrm{km})$
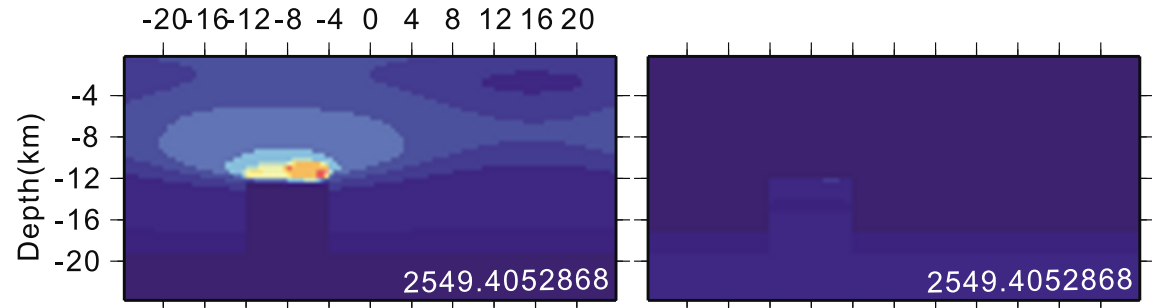

(b) Case3

$\mathrm{X}(\mathrm{km})$

$-20-16-12-8 \quad-4 \quad 0 \quad 4 \quad 8 \quad 121620$

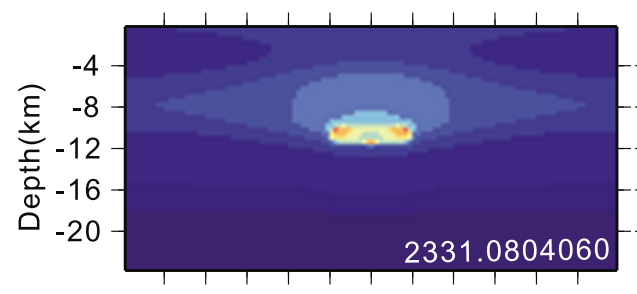

Slip velocity

due to flow $\log$ slip velocity $(\mathrm{m} / \mathrm{s})$

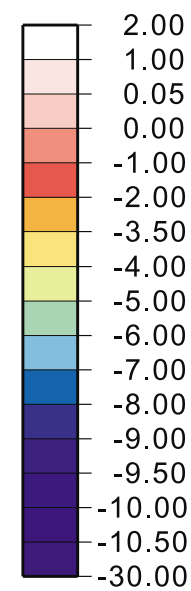

Fig. 5. Frictional slip velocity distribution (left) and slip velocity distribution due to flow (right) just before dynamic rupture for the case 2 (a), and case $3(\mathrm{~b})$. In case 2 , a low viscosity region is located at $-12(\mathrm{~km}) \leq x \leq-4(\mathrm{~km})$, and $z \geq 12(\mathrm{~km})$. In case 3 , a high-pore fluid pressure region is located at $-8(\mathrm{~km}) \leq x \leq 8(\mathrm{~km})$, and $z \geq 12(\mathrm{~km})$.

nucleation process is determined by the nonuniform distribution of the depth of the frictional-viscous transition zone. Our numerical results are consistent with observations. The depth variations of the frictional-viscous transition zone could be caused by a nonuniform thermal structure or heterogeneity in the pore fluid distribution or properties of rock involved. From actual observations of the exhumed fault zone, Shigematsu et al. (2003) found that a degree of ductile deformation changes along the frictional-viscous transition zone. Their observations indicate that there exist large depth variations of the frictional-viscous transition.

Shigematsu et al. (2003) also pointed out that a cataclasite zone coexists with a mylonite zone. In our model, dynamic rupture extends to the deeper part of the fault zone where viscous flow is dominant during interseismic period. Our numerical results suggest that coexistence of the mylonite zone and the cataclasite zone can occur.

\subsection{Constitutive law at high slip velocity for ductile material}

Iio et al. (2002) reported observations in which large precursory slips occurred below the deeper part of the seismogenic zone just before the main event. In the case of modeling where friction coexists with power law creep, we can reproduce both stationary aseismic slip below the deeper part of the seismogenic zone during the interseismic period and a local precursory slip associated with the nucleation process in the deeper part of the seismogenic zone just before the main event. However, we can not reproduce precursory slips that occur below the deeper part of the seismogenic zone before the main event. Therefore, to investigate precursory slips below the seismogenic zone, we need to consider other mechanisms. From the experimental study by Shimamoto (1986), we expect that, around the brittle-ductile transition zone, at very low velocity $\left(v \ll 10^{-6} \mathrm{~m} / \mathrm{s}\right)$ the flow process is dominant; at low velocity $\left(v \approx 10^{-6} \mathrm{~m} / \mathrm{s}\right), \tau_{s s}$ decreases with $v$; and at high slip velocity $\left(v \gg 10^{-6} \mathrm{~m} / \mathrm{s}\right), \tau_{s s}$ increases with $v$. To model slip acceleration below the deeper part of the seismogenic zone, we need to consider the frictional behavior from low slip velocity to high slip velocity: i.e. velocity-weakening at low slip velocity and velocity-strengthening at high slip velocity.

Shibazaki and Iio (2003) investigated a friction law that exhibits velocity-weakening at low slip velocity and velocity-strengthening at high slip velocity, following the model of static aging proposed by Estrin and Bréchet (1996). Shibazaki and Iio (2003) used the Dieterich-Ruina friction law with cut-off velocity (Okubo, 1989):

$$
\mu(\psi, v)=\mu_{*}+a \ln \left(v / v_{1}\right)+b \ln \left(v_{2} \psi / D_{c}+1\right)
$$

where $v$ is an instantaneous slip velocity, $v_{1}$ is a reference velocity to a direct effect, $v_{2}$ is a cut-off velocity to an evolution effect, $\psi$ is a state variable that characterizes the evolving state of the sliding surfaces, and $D_{c}$ is a characteristic displacement that scales the evolution of the state variable. In the Dieterich-Ruina friction law, a state variable can be written as

$$
d \psi / d t=1-\psi v / D_{c}
$$

In this friction law $d \mu_{s s} /(d \ln v)=a-b<0$ for $v \ll v_{2}$ but $d \mu_{s s} /(d \ln v)=a>0$ for $v \gg v_{2}$, where $\mu_{s s}(v)=$ $\mu_{*}+a \ln \left(v / v_{1}\right)+b \ln \left(v_{2} / v+1\right)$ is the steady state friction. Shibazaki and Iio (2003) obtained the numerical results showing that slow slip events occur several ten years before instability and that a slow precursory slip occurs in the deeper part of the seismogenic zone prior to instability. 
Kato (2003) also investigated a 2D fault model more precisely using a friction law that exhibits velocity weakening at low slip velocity and velocity strengthening at high slip velocity; they found that a slow precursory slip can occur below the deeper part of the seismogenic zone.

There are very few experimental studies investigating the constitutive law around the frictional-viscous transition (e.g. Masuda et al., 2002). Therefore, in future, we need to investigate this and to confirm that velocity strengthening due to flow at very low slip velocity, velocity weakening at low slip velocity and velocity strengthening at high slip velocity can occur.

\section{Conclusions}

We considered a model of earthquake generation processes using a constitutive law combining friction and flow law. By using this law, we can represent the fault behavior in which frictional slip coexists with the flow process at the frictional-viscous transition zone. We considered the limited region where viscous deformation is high along the frictional-viscous transition zone, and investigate the role of this region in the nucleation process. This large amount of slip due to flow at low-viscosity region during the interseismic period is thought to cause stress to concentrate in the surrounding regions, and nucleation starts just above the low-viscosity region. We also investigated a case in which a region of high pore-fluid pressure exists in the deeper part of the seismogenic zone. If there is a region of high porefluid pressure in the deeper part of the seismogenic zone, the depth of the brittle-viscous transition zone is thought to increase. Nucleation zone is located just above the region of high pore-fluid pressure. We found that the location of the nucleation process is determined by the nonuniform distribution of the depth of the frictional-viscous transition zone. In future we need to model fault slip processes using a constitutive law investigated by experimental studies under conditions close to those of the brittle-viscous transition.

Acknowledgments. We would like to thank Koichiro Fujimoto, Yoshihisa Iio, Koji Masuda, and Takashi Arai for their useful discussions about this study. We also thank two reviewers for useful comments. This study was supported by a grant from the Comprehensive Joint Research of the Special Coordination Funds for Promoting Science and Technology, for "Comprehensive Research on Slip and Flow Processes in and below the Seismogenic Region" in Japan.

\section{References}

Chester, F. M., A rheologic model for wet crust applied to strike-slip faults, J. Geophys. Res., 100, 13,033-13,044, 1995.

Estrin, Y. and Y. Brchet, On a model of frictional sliding, Pageoph., 147, 1996.

Garatani, K., B. Shibazaki, Y. Iio, T. Sagiya, and H. Okuda, FEM modeling crustal deformation with non-linear visco-elasticity, plasticity, and faulting, Earth Planets Space, 2005 (submitted).
Iio, Y. and Y. Kobayashi, Is the plastic flow uniformly distribted below the seismogenic region?, Earth Planets Space, 54, 1085-1090, 2002.

Iio, Y., Y. Kobayashi, and T. Tada, Large earthquakes initiated by the acceleration of slips on the downward extensions of seismogenic faults, Earth Planet. Sci. Lett., 202, 337-343, 2002.

Ito, K., Regional variations of the cutoff depth of seismicity in the crust and their relation to heat flow and large inland earthquakes, J. Phys. Earth, 38, 223-250, 1990.

Iwasaki, T. and R. Sato, Strain field in a semi-infinite medium due to an inclined rectangular fault, J. Phys. Earth, 27, 285-314, 1979.

Kato, N., A possible model for large preseismic slip on a deeper extension of a seismic rupture plane, Earth Planet. Sci. Lett., 216, 17-25, 2003.

Kato, N. and T. Hirasawa, A numerical study on seismic coupling along subduction zones using a laboratory-derived friction law, Phys. Earth Planet. Inter., 102, 51-68, 1997.

Marone, C., Laboratory-derived frictional laws and their application to seismic faulting, Annu. Rev. Earth Planet. Sci., 94, 12,321-12,335, 1998.

Masuda, K., K. Fujimoto, and T. Arai, A new gas-medium, high-pressure and high-temperature deformation apparatus at AIST, Japan, Earth Planets Space, 54, 1091-1094, 2002.

Nakatani, M., Conceptual and physical clarification of rate and state dependent friction law: Thermally activated rheology of frictional sliding, J. Geophys. Res., 106, 13,347-13,380, 2001.

Okubo, P. G., Dynamic rupture modeling with laboratory-derived constitutive relations, J. Geophys. Res., 94, 12,321-12,335, 1989.

Poirier, J. P., Creep of Crystals, Cambridge University Press, Cambridge, 1985.

Reinen, L. A., Slip styles in a spring-slider model with a laboratory-derived constitutive law for serpentinite, Geophys. Res. Lett., 27, 2037-2040, 2000.

Rice, J. R., Spatio-temporal complexity of slip on a fault, J. Geophys. Res., 98, 9885-9907, 1993.

Scholz, C. H., The Mechanics of Earthquakes and Faulting, Cambridge University Press, 1990.

Shibazaki, B. and Y. Iio, On the physical mechanism of silent slip events along the deeper part of the seismogenic zone, Geophys. Res. Lett., 30, 2003GL017047, 2003.

Shibazaki, B., H. Tanaka, H. Horikawa, and Y. Iio, Modeling slip processes at the deeper part of the seismogenic zone using a constitutive law combining friction and flow laws, Earth Planets Space, 54, 1211-1218, 2002.

Shigematsu, N., K. Fujimoto, T. Ohtani, H. Tanaka, Y. Miyashita, and T. Tomita, Structures of fault zones in the brittle-plastic transition zone of the continental earth's crust: A case study of the Hatagawa fault zone, Journal of Geography, 112, 897-914, 2003 (in Japanese with English abstract).

Shimamoto, T., A transition between frictional slip and ductile flow undergoing large shearing deformation at room temperature, Science, 231, 711-714, 1986.

Tanaka, H., B. Shibazaki, N. Shigematsu, K. Fujimoto, T. Ohtani, Y. Miyashita, T. Tomita, K. Omura, Y. Kobayashi, and J. Kameda, Growth of plastic shear zone and its duration inferred from theoretical consideration and observation of an ancient shear zone in the granitic crust, Earth Planets Space, 54, 1207-1210, 2002.

Tse, S. T. and J. R. Rice, Crustal earthquake instability in relation to the depth variation of frictional slip properties, J. Geophys. Res., 91, 94529472, 1986.

Zhao, D., H. Kanamori, and H. Negishi, Tomography of the source area of the 1995 Kobe earthquake: Evidence for fluids at the hypocenter?, Science, 274, 1891-1894, 1996.

B. Shibazaki (e-mail: bshiba@kenken.go.jp), N. Shigematsu, and H. Tanaka 